\title{
Graphical user interface for input output characterization of single variable and multivariable highly nonlinear systems
}

\author{
M. D. Shahrukh Adnan Khan ${ }^{1,2}$, Sharsad Kara Kuni ${ }^{2}$, G. K. M. Sadikul Amin ${ }^{3}$, Mukul Agarwal ${ }^{4}$ \\ ${ }^{1}$ Assistant Professor, University of Asia Pacific, Dhaka, Bangladesh \\ ${ }^{2}$ University of Nottingham, JalanBroga, Selangor, Malaysia \\ ${ }^{3}$ Director, Epic Airways Limited, Dhaka, Bangladesh \\ ${ }^{4}$ Managing Director, MukTar Enterprises Pte Ltd, 320 Serangoon Road, 4-46 Serangoon Plaza, Singapore
}

\begin{abstract}
This paper presents a Graphical User Interface (GUI) software utility for the input/output characterization of single variable and multivariable nonlinear systems by obtaining the sinusoidal input describing function (SIDF) of the plant. The software utility is developed on MATLAB R2011a environment. The developed GUI holds no restriction on the nonlinearity type, arrangement and system order; provided that output(s) of the system is obtainable either though simulation or experiments. An insight to the GUI and its features are presented in this paper and example problems from both single variable and multivariable cases are demonstrated. The formulation of input/output behavior of the system is discussed and the nucleus of the MATLAB command underlying the user interface has been outlined. Some of the industries that would benefit from this software utility includes but not limited to aerospace, defense technology, robotics and automotive.
\end{abstract}

\section{Introduction}

Mathematical model is a prerequisite in most of the cases for a controller to begin a design. In real life systems, mathematical models are quite sophisticated and most of the times it could be represented only by using computer codes [1]. With the fact that all practical real life systems contain nonlinearities, describing function approach has attracted considerable interest when conventional controller design techniques were not able to satiate the designers. Though this paper is not intended to discuss on the limitations and advantages of describing function method, it is desirable to point out a few of them. The primary motivation of the use of describing functions is to extend the very powerful transfer function approach of linear systems to nonlinear systems [2]. One of the most important characteristics of nonlinear system is the dependency on the amplitude of excitation. This dependency on amplitude of nonlinear plant can be well represented via the sinusoidal-input describing function model of the plant [3]. In general, most of the models can be presented in the following form;

$$
\begin{aligned}
& \dot{x}=f(x, u, t) \\
& Y=g(x, u, t)
\end{aligned}
$$

where $x$ is the state vector of dimension $n, u$ is the input vector of dimension $m, y$ is the output vector of dimension $l$, and $t$ represents the time.
Since numerous literatures have been produced discussing the describing function model concept, only some of the key points underlying this approach will be reviewed here. A describing function can be defined as the ratio of the fundamental component of the output of a nonlinear process to the sinusoidal input signal $[2,4]$. With the modern formulation of SIDF modelling (Taylor (1983), Taylor and Strobel (1985), Nassirharand (1987)), based on evaluating 'Fourier Integrals' by integrating the equations of motion, most of the earlier restrictions such as the requirement of sinusoidal input to each nonlinearity are lifted. The only assumption left with the describing function model is that it forms a better representation of the nonlinear system. However, an input/output formulation of following general framework is obtained using this approach [3];

$$
\begin{gathered}
x=X \sin \omega t \\
y \approx a \operatorname{Re}[G(j \omega, a) e j \omega t]
\end{gathered}
$$

\section{Application of nonlinear control system}

To put in layman's words, the input signals are more often towards minimum and maximum values than in the middle [1]. Thus, need for control design techniques, where robustness as the primary design criterion is imposed. It has been well established that sinusoidal input describing function models provides an excellent 
platform for robust control system design due to the fact that no other conventional design techniques exist so far; that may attribute to its ability to capture the amplitude sensitive characteristics of nonlinear plant [1] [3-6]. In this work, particular interest will be on formulation of input/output behaviour of such industrial areas as aerospace, defence and robotics where the amplitude distribution of excitation signals are similar to sinusoidal case. A 3 seat, piston engine based, fixed wing British light aircraft was designed by Mr. John Edgily [7]. It first flew on 14th December 1979, achieving certification in the year of 1985 , on February 8 . With a visibility of a Helicopter and stability of a fixed wing, the aircraft namely OPTICA is happened to be much safer and costeffective [8]. With a stall speed of $108 \mathrm{~km} / \mathrm{hr}$, service ceiling of $4275 \mathrm{~m}$ and range of $1056 \mathrm{~km}$, the carries focuses dual control capability for pilot training. The proprietor of the architectural design of carrier Optica, MukTar Enterprises, proposed to establish a nonlinear control system for their aircraft [9]. Aircraft flight control design is conventionally created on linear control principle. However, to reach tactical benefits, modern aircrafts struggle towards performing exercises outside the region in which the dynamics of flight are nonlinear. Hence, the necessity for nonlinear tools arises.

Figure 1 and 2 shows the proposed set-up spot for establishing a GUI based nonlinear controlling system in carrier Optica.

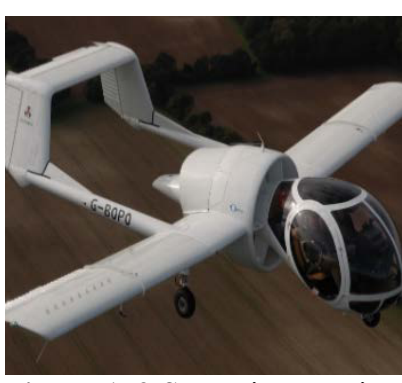

Figure 1. 3 Seat, Piton Engine Fixed Wing OPTICA.

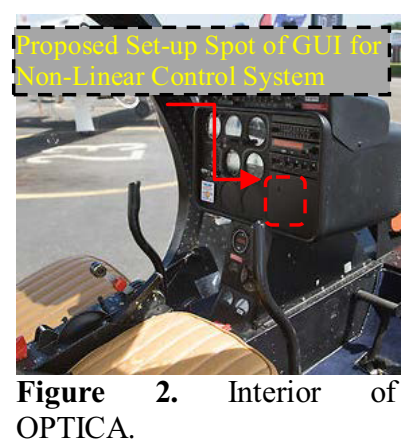

\section{Graphical user interface}

A Graphical User Interface (GUI) has been developed in the MATLAB environment which would enable the user to derive the input/output characterization of highly nonlinear single variable and multivariable nonlinear systems through generating Sinusoidal input describing function models of the plant. The GUI shown in Fig. 3 is designed for MATLAB R2011a platform. The main utility of the GUI are 1) generate the SIDF model from the state-variable models 2) output the pseudo frequency bode plots 3) automate time-data plots. The User Interface has no restriction on nonlinearity type provided system output(s) for input(s) must be obtainable by either simulation or experiments.

Apart from the user friendly environment it provides, the developed GUI also allows user to save the SIDF developed each time for different desired set of operational conditions and can obtain a combined pseudo bode plots which may be used for comparing the system behaviour under different excitation frequencies and amplitudes. The other features enable the user to save the plots and output data which may be retrieved for later use. The software utility may be better demonstrated by an example from both single-variable and multi-variable case.

\section{Single-Input Single-Output Case}

Before demonstrating an example problem, it is worthwhile to discuss some of the important input/output characterization techniques of single variable system followed by an insight to the user interface environment. The formulation of input/output characterization technique for single variable is based on Nassirharand's work which was originally based on Taylor's Fourier's analysis approach to develop the sinusoidal input describing function models [3]. The plant is excited by the sinusoidal input of the following form.

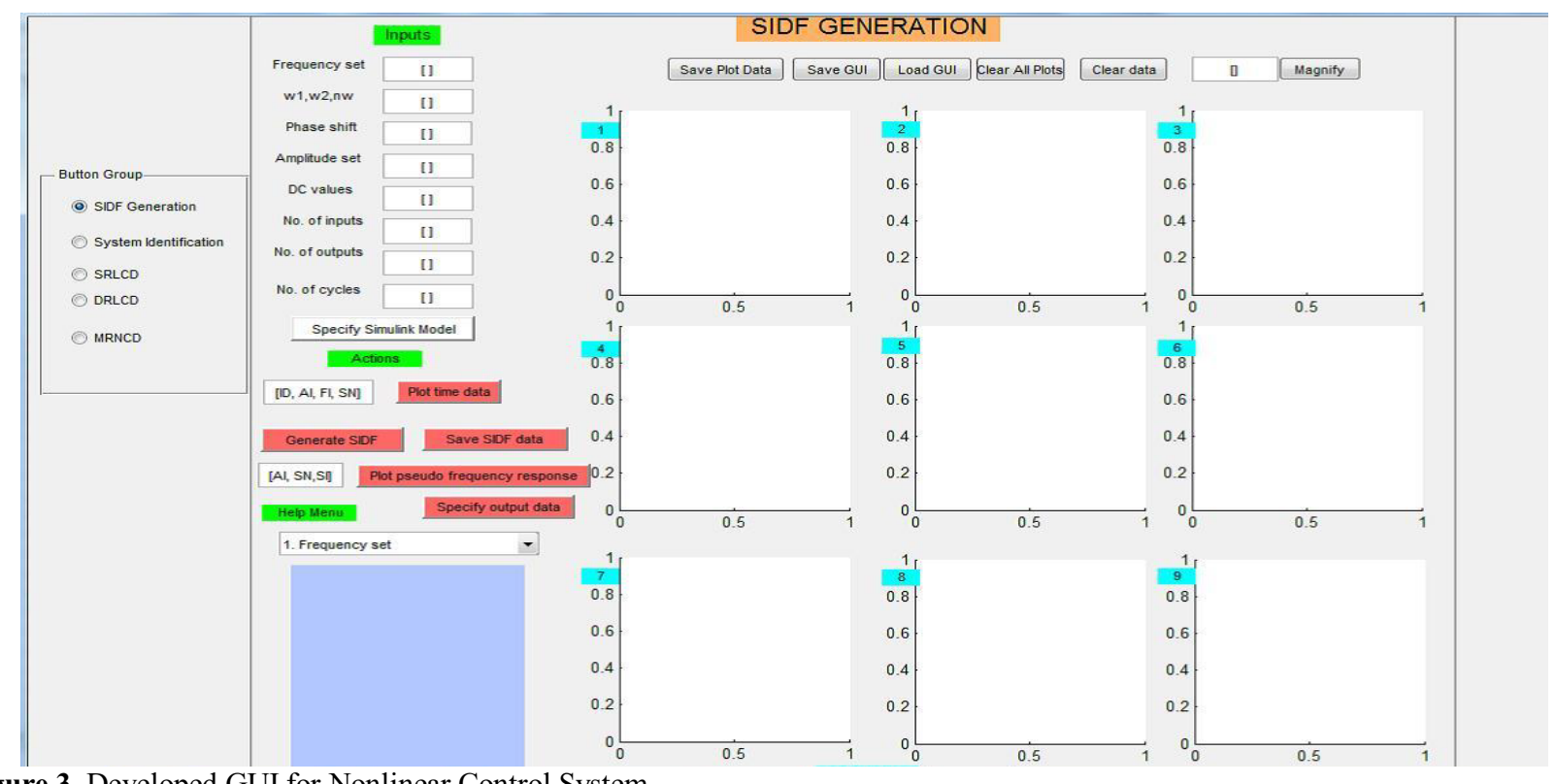

Figure 3. Developed GUI for Nonlinear Control System. 


$$
u(t)=u_{0}+a \cos (\omega t)
$$

Here, $\mathrm{u}$ is the input to the system, $\mathrm{u} 0$ is the $\mathrm{DC}$ value of the input signal, a is the amplitude of the excitation and $\omega$ the frequency of the excitation signal. The approach adapted herein, compute the output $y(t)$ as a function of time by numerically integrating the equations of motion. Fourier integrals of the following form are evaluated once $y(t)$ approaches steady state.

$$
I_{m, k}=\int_{(k-1) t}^{k t} y(t) e^{-j m \omega t} d t
$$

Here, period index $=1,2,3 \ldots . .$, harmonic index $=0,1,2 \ldots$, and period $\mathrm{T}$ is given by $2 \pi / \omega$. The $\mathrm{DC}$ component of the response is $\mathrm{I}_{0, \mathrm{k}}$ and the pseudo transfer function is given as follows.

$$
\mathrm{G}_{\mathrm{m}, \mathrm{k}}\left(\mathrm{jm} \omega, \mathrm{u}_{0}, \mathrm{a}\right)=\omega \mathrm{I}_{\mathrm{m}, \mathrm{k}} / \mathrm{a} \pi .[3]
$$

The GUI requires the user to input the following parameters and file(s) in order to obtain the corresponding sinusoidal input describing function models of the nonlinear plant.

(1) Either desired frequency array in $\mathrm{rad} / \mathrm{sec}$ or range of the excitation frequency of interest.

(2) An array of desired excitation amplitude set.

(3) For single variable case, phase shift values must not be entered.

(4) An array of DC values of the input sinusoids. The size of this vector must be equal to the size of input amplitudes.

(5) The number of inputs and outputs should be entered as 1.

(6) Desired Fourier integration cycles for each frequency range in the ascending order.

(7) Simulink file corresponding to the system for which SIDF model to be generated.

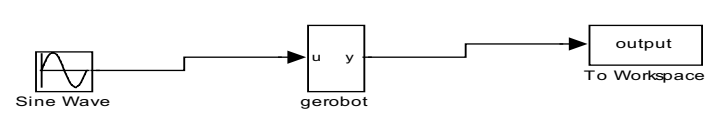

Figure 4. Block Diagram for Single variable Case.

In many cases where mathematical models are not available, the user may want to generate sinusoidal input describing model where system output(s) is obtained from experimental results. The GUI presented also can be well used with such conditions where Simulink file is not required. In such cases, the user must provide the output data in a prescribed format where first column is time, the second column is the first output, and the third column is second output (in case of multivariable) and so on. The nucleus of the underlying software of the GUI is a MATLAB function that generates the real and imaginary parts of the describing function models as a function of excitation frequencies and amplitudes. Consider an example problem of the sort encountered in robotics. Figure 4 represents the user input Simulink file for the nonlinear single variable plant. We may obtain the SIDF models of the single-input single-output system around the following operating conditions for this example.(1) excitation frequencies of [ $\left[\begin{array}{lll}1 & 2 & 3\end{array}\right] ;(2)$ excitation amplitudes of [3 4 5 5];(3) DC values of [0 00 0];(4) NCYCLE inputs array covering the frequency range as $\left[\begin{array}{lllll}1 & 2 & 3 & 3 & 5\end{array}\right]$. Once the desired values are entered, the user may generate the SIDF model using the GUI. The software utility will output the sinusoidal input describing function as gr and gi (see Appendix 1). The software will also generate the pseudo frequency bode plots which are shown in Fig.6 as well as time-data plots shown in Fig. 5. Time-data plots can be analyzed in row wise to see that the first plot corresponds to all amplitudes and all corresponding frequencies and go through maximum NCYCLE value (that is 5 for this example problem). The second graph for time-data plot represents the first frequency and it cover through its Fourier integral cycle range of 3. Similar analysis can be done for plot 3 and 4 which constitute for second and third frequencies plotting through their NCYCLE values respectively.
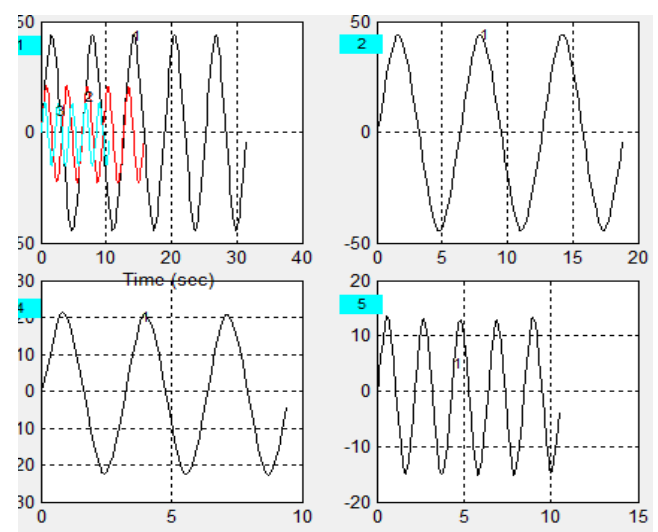

Figure 5. Time-Data Plots (Single Variable).
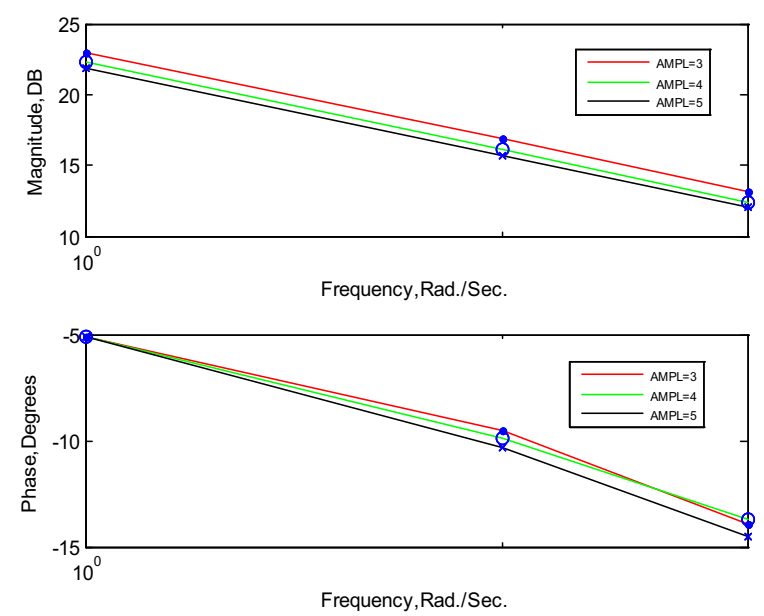

Figure 6. PSEUDO-BODE Plots (Single Variable).

\section{Multi-input multi-output case}

Let us discuss some of the main input/output characterization techniques involved in the case of multiinput multi-output system as well as GUI environment for multivariable plant before coming to the example problem. For multivariable case, one cannot be able to determine the effects of each input to the corresponding 
outputs; hence the principle of superposition is not valid. Furthermore, it has to be noted that the separate effects of each input on each output cannot be determined if any of the input frequencies are same. Keeping these points in mind, the input/output behavior of a multivariable system can be formulated by assuming the input to the system is of following general form [1].

$$
u p(t)=u 0, p+a p \cos (\omega p t+\theta p) ; \quad p=1,2 \ldots . m
$$

Here, $u$ is the input to the system, $\mathrm{u} 0$ is the $\mathrm{DC}$ value of the input signal, a and $\omega$ are the excitation amplitude and frequency respectively, $\theta$ is the phase shift and $p$ is the input channel index. Output as a function of time, $y_{q}(t)$ is obtained by numerically integrating the equations of motion and thus the Fourier integral matrix, $I_{q, p}^{h, k}$ are evaluated as below.

$$
I_{q, p}^{h, k}=\int_{(k-1) t}^{k t} y_{q}(t) \exp \left(-j h\left(\omega_{p} t+\theta_{p}\right) d t\right.
$$

Here $\mathrm{h}$ is the harmonic index, $\mathrm{k}$ the period index, $\mathrm{q}$ the output channel index and $\mathrm{T}$ is the overall period. The pseudo transfer function $\left(G_{q, p}^{1, k}\right)$ is given as follows.

$$
G_{q, p}^{1, k}\left(j \omega_{q} ; u_{0}, a, \theta\right)=\left(2 / a_{p} T\right) I_{q, p}^{1, k}
$$

The convergence criteria for Fourier Integrals are as follows;

$$
\begin{gathered}
\left|\frac{M_{q, p}^{k}-M_{q, p}^{k-1}}{M_{q, p}^{k}}\right|<\varepsilon_{M} \\
\left|\phi_{q, p}^{k}-\phi_{q, p}^{k-1}\right|=\varepsilon_{\varphi}
\end{gathered}
$$

Here, $M_{q, p}^{k}$ is the log magnitude and $\phi_{q, p}^{k}$ is the phase of $G_{q, p}^{k}$ [1]. In order to obtain the sinusoidal input describing function models and thus the corresponding pseudo bode plots and time data plots, the user needs to input all the required parameters as discussed above in the case of single-input single-output system. Those are not repeated here except some of the points peculiar to the multivariable case.1) the number of rows of frequency array input must equals the number of inputs. 2) the amplitude array column size must be same as the number of inputs. 3) Unlike single variable case, user must enter an array containing phase shift values and the size of this matrix has to be identical to the size of frequency input matrix. 4) the number of inputs corresponding to the multivariable plant and the desired number of outputs need to be provided by the user .5) Fourier integral cycle input must cover all frequencies of first channel (or first row of frequency matrix). The rest of the requirements would remain unchanged. The key MATLAB command takes the excitation amplitude array and excitation frequency array as inputs and its main outputs are the real and the imaginary parts of the describing function models (pseudo frequency response). The other outputs are the value of gain error convergence parameter and the value of phase error convergence parameter.

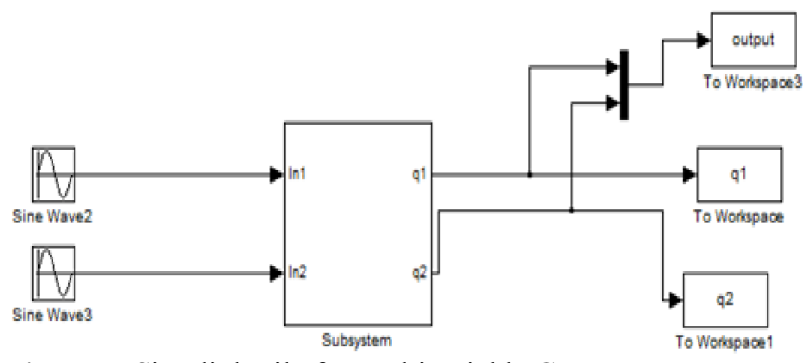

Figure 7. Simulink File for Multivariable Case.

\section{Simulink file for multivariable case}

Consider the nonlinear multi-input multi-output plant with two inputs as shown in Fig. 7 which represents the user-input Simulink file. For the example purpose, the pseudo frequency response data(SIDF function) of the system can be obtained for the following input values: 1) excitation array frequency values of $\left[\begin{array}{llll}0.1 & 0.2 & 0.3 ; 0.4 & 0.5\end{array}\right.$ $0.6]$; 2)array of phase shift values is given by [pi/2 pi/2 $\mathrm{pi} / 2 ; \mathrm{pi} / 2 \mathrm{pi} / 2 \mathrm{pi} / 2]$; 3)excitation amplitude set is given by [7 8]; 4) DC value array of [0 0]; 5)number of inputs and outputs are entered as $2 ; 6$ )Input for No. of cycles are $\left[\begin{array}{lllll}0.1 & 0.2 & 2 & 0.3\end{array}\right]$. At this stage, the user may execute the software utility for generating the describing function. The pseudo-bode plots for the given example generated using the GUI is shown in Fig. 8 and time-data plots can be seen from Fig. 9. Similar analysis as in the case of single-input single-output can be done here for time-data figures to see how different frequencies for its corresponding Fourier integral cycles are plotted for time-data analysis.
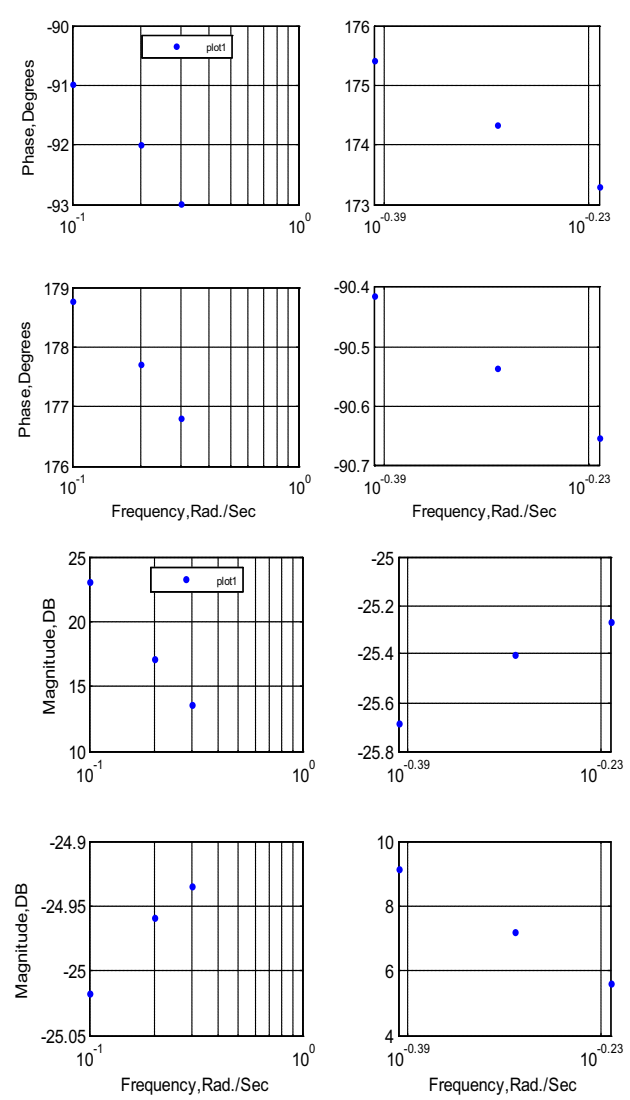

Figure 8. PSEUDO-BODE Plots for Multivariable. 


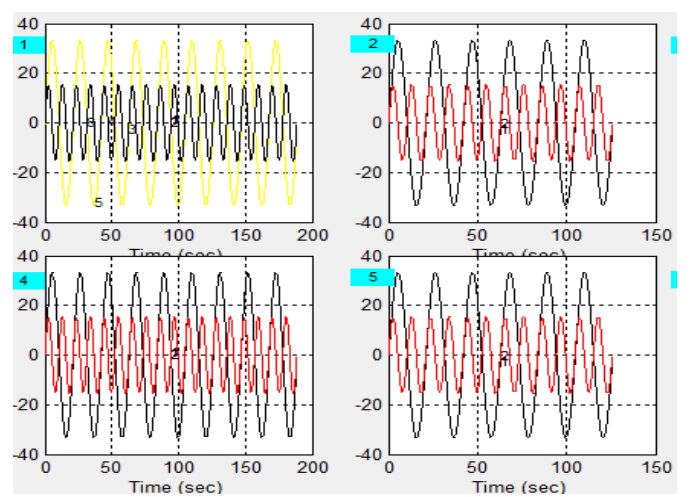

Figure 9. TIME-DATA Plots for Multivariable.

\section{Conclusions}

The proposed objective of the study was to develop a Graphic user interface utility for developing sinusoidal input describing function for both single variable and multivariable nonlinear systems. The aim was achieved. The formulation of input/output characterization is based on Fourier analysis where equations of motion are integrated to obtain the Fourier integrals. This procedure is repeated for different frequencies and amplitudes to obtain the sinusoidal input describing function model of the plant. Once the SIDF models are generated, system characteristics can be improved using conventional control design theories [3]. As a future work, the development of a user interface utility for the design of single range controller design for both single-input single-output case and multivariable case using describing function model can be proposed. With higher degree non-linear equations, the future calibration with GUI could be a realistic control system to work in the aircrafts. Implementing this proposed GUI interface for nonlinear controlling system in Optica, we can possibly find some credible features of characteristics in controlling modality of Optica flying Nonlinear equations of motion in aircraft; for example computation of exact flight paths with motions detection, optimization of flight paths along with nonlinear, time-varying large amplitude motions and significant change in mass in the aircraft could be a future scope in this newly developed interface considering higher degree non-linear equations.

\section{References}

1. Nassirharand, A. Karimi, H. 2002, Input/output characterization of highly nonlinear multivariable systems, AdvEngng software 33(2002).

2. Shinners Stanley, Describing function concept, Chap.5 section 5.7 of advanced modern control system theory and design, pp. 334-36, 1998.

3. Nassirharand, Input/output characterisation of highly nonlinear systems, .AdvEngng software, 6(3), pp. 129-33, 1987.

4. Nagrath, I. J. Gopal, The describing function method: Basic concepts. Chap.15 section 15.7 of control Systems Engineering, Fifth Edition, pp. 742-44, 2010.

5. Nassirharand, Design of dual-range linear controllers for nonlinear systems. ASME Trans DynSyst, Measure, Control, 1991.

6. Taylor JH, Strobel KL, Applications of a nonlinear controller design approach based on quasilinear system models, Proceedings of American Control Conference. San Diego, pp. 817, 1984.

7. Ola H"arkegard and S. Torkel Glad, A backstepping design for flight path angle control. In Proceedings of the 39th Conference on Decision and Control, pages 3570-3575, Sydney, Australia, December 2000.

8. Ola H"arkegard and S. Torkel Glad, Flight control design using backstepping. Technical Report LiTHISY-R-2323, Department of Electrical Engineering, Link"opingsuniversitet, Sweden, December 2000.

9. WWW.flyoptica.com 